\title{
Novel targeting using nanoparticles: an approach to the development of an effective anti-leishmanial drug-delivery system
}

This article was published in the following Dove Press journal:

International Journal of Nanomedicine

18 February 2014

Number of times this article has been viewed

\author{
Tatiana G Ribeiro' \\ Miguel A Chávez-Fumagalli \\ Diogo G Valadares ${ }^{3}$ \\ Juçara R França' \\ Lívia B Rodrigues' \\ Mariana C Duarte ${ }^{2}$ \\ Paula S Lage ${ }^{2}$ \\ Pedro H R Andrade 4 \\ Daniela P Lage ${ }^{4}$ \\ Leonardo $\vee$ Arruda $^{5,6}$ \\ Daniel R Abánades ${ }^{5,6}$ \\ Lourena E Costa ${ }^{2}$ \\ Vivian T Martins ${ }^{3}$ \\ Carlos AP Tavares ${ }^{3}$ \\ Rachel O Castilho ${ }^{1,7, *}$ \\ Eduardo AF Coelho 2,4,* \\ André AG Faraco $1,7, *$ \\ 'Programa de Pós-Graduação em Ciências \\ Farmacêuticas, Faculdade de Farmácia, \\ Universidade Federal de Minas Gerais, Belo \\ Horizonte, Minas Gerais, Brazil; ${ }^{2}$ Programa de \\ Pós-Graduação em Ciências da Saúde: Infectologia \\ e Medicina Tropical, Faculdade de Medicina, \\ Universidade Federal de Minas Gerais, Belo \\ Horizonte, Minas Gerais, Brazil; ${ }^{3}$ Departamento \\ de Bioquímica e Imunologia, Instituto de Ciências \\ Biológicas, Universidade Federal de Minas \\ Gerais, Belo Horizonte, Minas Gerais, Brazil; \\ ${ }^{4}$ Departamento de Patologia Clínica, COLTEC \\ Universidade Federal de Minas Gerais, Belo \\ Horizonte, Minas Gerais, Brazil; ${ }^{5}$ Programa de Pós- \\ Graduação em Patologia Humana, Universidade \\ Federal da Bahia, Salvador, Bahia, Brazil; ${ }^{6}$ Centro \\ de Pesquisas Gonçalo Moniz (CPqGM), Fundação \\ Oswaldo Cruz (FIOCRUZ), Salvador, Bahia, Brazil; \\ ${ }^{7}$ Departamento de Produtos Farmacêuticos, \\ Faculdade de Farmácia, Universidade Federal de \\ Minas Gerais, Belo Horizonte, Minas Gerais, Brazil \\ *These authors contributed equally to \\ this work
}

Correspondence: Eduardo AF Coelho

Laboratório de Biotecnologia Aplicada ao Estudo das Leishmanioses, Departamento de Patologia Clínica, COLTEC, Universidade Federal de Minas Gerais, Avenida Antônio Carlos, 6627,

31 270-901, Belo Horizonte, Minas Gerais, Brazil Tel/Fax +55 3l 34094983

Email eduardoferrazcoelho@yahoo.com.br
Abstract: The study reported here aimed to develop an optimized nanoparticle delivery system for amphotericin B (AmpB) using a polyelectrolyte complexation technique. For this, two oppositely charged polymers presenting anti-leishmanial activity - chitosan (Cs) and chondroitin sulfate $(\mathrm{ChS})$ - were used: $\mathrm{Cs}$ as a positively charged polymer and $\mathrm{ChS}$ as a negatively charged polymer. The chitosan (NQ) nanoparticles, chitosan-chondroitin sulfate (NQC) nanoparticles, and chitosan-chondroitin sulfate-amphotericin B (NQC-AmpB) nanoparticles presented a mean particle size of 79, 104, and $136 \mathrm{~nm}$, respectively; and a polydispersity index of 0.2 . The measured zeta potential of the nanoparticles indicated a positive charge in their surface, while scanning and transmission electron microscopy revealed spherical nanoparticles with a smooth surface. Attenuated total reflectance-Fourier transform infrared spectroscopy analysis showed an electrostatic interaction between the polymers, whereas the release profile of AmpB from the NQC-AmpB nanoparticles showed a controlled release. In addition, the Cs; $\mathrm{ChS}$; and NQ, NQC, and NQC-AmpB nanoparticles proved to be effective against promastigotes of Leishmania amazonensis and Leishmania chagasi, with a synergistic effect observed between $\mathrm{Cs}$ and $\mathrm{ChS}$. Moreover, the applied NQ, NQC, and NQC-AmpB compounds demonstrated low toxicity in murine macrophages, as well as null hemolytic activity in type $\mathrm{O}^{+}$human red blood cells. Pure AmpB demonstrated high toxicity in the macrophages. The results show that cells infected with L. amazonensis and later treated with $\mathrm{Cs}$, ChS, NQ, NQC, NQC-AmpB nanoparticles, or pure $\mathrm{AmpB}$ presented with a significant reduction in parasite number in the order of $24 \%, 31 \%, 55 \%$, $66 \%, 90 \%$, and $89 \%$, respectively. The data presented indicate that the engineered NQC-AmpB nanoparticles could potentially be used as an alternative therapy to treat leishmaniasis, mainly due its low toxicity to mammals' cells.

Keywords: chitosan, chondroitin, amphotericin B, nanoparticles, Leishmania spp.

\section{Introduction}

Leishmaniasis is a disease with a wide spectrum of clinical manifestations caused by different species of protozoa belonging to the Leishmania genus. ${ }^{1}$ The disease has high rates of morbidity and mortality throughout the world. There are 350 million people in 98 countries at risk of contracting the disease, ${ }^{2}$ and approximately 700,000 to 1.2 million cases of tegumentary leishmaniasis, and 200,000 to 400,000 cases of visceral leishmaniasis are registered annually worldwide. ${ }^{3}$

The parenteral administration of pentavalent antimony compounds continues to be the first treatment of choice; however, the occurrence of side effects, such as anorexia, myalgias, arthralgias, chemical pancreatitis, leucopenia, and cardiotoxicity, is an important problem reported by patients. ${ }^{4,5}$ Amphotericin B (AmpB), a secondline drug, is a highly hydrophobic antifungal product with effective anti-leishmanial 
activity, but its clinical use is limited by high toxicity. ${ }^{6,7}$ To improve the therapeutic index of $\mathrm{AmpB}$ and to reduce its cytotoxicity, lipid-based formulations have been developed for parenteral administration, such as AmBisome ${ }^{\circledR}$ (Gilead Sciences, Inc., Foster City, CA, USA), Amphocil ${ }^{\circledR}$ (Kadmon Pharmaceuticals, New York, NY, USA), and Abelcet ${ }^{\circledR}$ (Sigma-Tau Pharmaceuticals, Inc., Gaithersburg, MD, USA). The World Health Organization has recommended the use of liposomal AmpB (L-AmpB) based on its high efficacy and safety. ${ }^{2,8}$ Despite improvements in therapeutic indexes for these lipid formulations, their use still remains limited due mainly to their high cost. ${ }^{9}$

Therefore, the development of new drug-delivery systems to treat leishmaniasis could be considered relevant. ${ }^{10}$ In this regard, a wide variety of techniques is available to produce polymeric nanoparticles, including solvent evaporation, interfacial polymerization, and emulsion polymerization methods. ${ }^{11}$ In relation to delivery systems using AmpB, de Carvalho et al developed a system containing AmpB encapsulated in poly(lactic-co-glycolic acid) and dimercaptosuccinic acid nanoparticles, ${ }^{12}$ Asthana et al formulated nanometric AmpB-encapsulated chitosan (Cs) nanoparticles using a polymeric deposition technique mediated by nanoemulsion template fabrication, ${ }^{13}$ Shao et al designed polymeric micelles using a formulation of 1,2-distearoylglycerol-3-phosphoethanolamine-N-[methoxy(polyethylene glycol)-2000]-based micelles loaded with AmpB,${ }^{14}$ and Yang et al formulated polymeric micelles prepared from a series of poly(ethylene glycol)-poly(lactide) co-polymers with various polylactide chain lengths for AmpB. ${ }^{15}$ Unfortunately, such examples frequently require the use of organic solvents or heat, which are undesirable steps and that may affect the integrity of the macrolide substances. ${ }^{11,16}$

Polyelectrolyte complexes (PECs) are formed by interactions between two macromolecules bearing oppositely charged groups. The encapsulation of a drug during the formation of PECs has shown great promise for use as drug-delivery carriers. ${ }^{17}$ Recently, PECs prepared from natural macromolecules have attracted attention for use in the development of drug-delivery systems, such as the complex between Cs and heparin, which have been formulated as vaccine adjuvants. ${ }^{18}$ Some studies have investigated the drug-delivery systems based on Cs-ChS complexes, such as Huang et al, ${ }^{19}$ Ganza-Gonzáles et $\mathrm{al},{ }^{20}$ and Sui et al, ${ }^{21}$ who have reported the use of Cs-ChS-based microcapsules for controlled release by 5-fluorouracyl, heparin, and metoclopramide, respectively, in their studies. Yeh et al characterized protein-loaded Cs-ChS nanoparticles using the PEC technique, ${ }^{22}$ while $\mathrm{Hu}$ et al studied the influence of charge on fluorescein isothiocyanate (FITC)-bovine serum albuminloaded Cs-ChS nanoparticles upon cell uptake in human Caco- 2 cell monolayers. ${ }^{23}$ Tsai et al produced doxorubicinencapsulated $\mathrm{Cs}-\mathrm{ChS}$ nanoparticles that were applied against cancer cells. ${ }^{24}$ However, to our knowledge, our study marks the first time the construction of a Cs-ChS based system for AmpB delivery has been developed for the treatment of leishmaniasis.

“Cs," b-(1,4)-2-amino-2-deoxy-D-glucan, prepared by deacetylation from chitin, presents many interesting properties, including biocompatibility, biodegradability, mucoadhesivity, and bioactivity. ${ }^{25} \mathrm{Cs}$ has an intrinsic pKa near 6.5 with a maximum of one positive charge per residue. 26 " $\mathrm{ChS}$ " is a glycosaminoglycan present in the extracellular matrix of cartilage that is used in the treatment of osteoarthritis and demonstrates an anti-inflammatory activity. ${ }^{27-29} \mathrm{ChS}$ is also a component of the dermal layer of the US Food and Drug Administration-approved skin substitute, ${ }^{30}$ commonly used to enhance re-epithelialization without causing scarring. ${ }^{31,32}$ Moreover, $\mathrm{ChS}$ can be degraded by colonic microflora, which can be degraded by colonic microflora. ${ }^{32,33}$

The purpose of the study presented here was to develop a nanoparticle system containing $\mathrm{Cs}, \mathrm{ChS}$, and $\mathrm{AmpB}$, and to evaluate its anti-leishmanial activity against in vitro promastigote and in vivo amastigote forms of Leishmania amazonensis and Leishmania chagasi. Studies were also performed to its minimum inhibitory concentration (half maximal inhibitory concentration [CC50]), as well as to verify the efficacy of pure $\mathrm{Cs}$ and $\mathrm{ChS}$, as well as chitosan (NQ), chitosan-chondroitin sulfate (NQC), and chitosanchondroitin sulfate-amphotericin B (NQC-AmpB) nanoparticles in the treatment of infected macrophages, in an attempt to develop an effective AmpB delivery system that would decrease the toxicity of this drug in mammals' cells, as well as produce an alternative therapeutic option to treat leishmaniasis.

\section{Materials and methods Preparation of NQ, NQC, and NQC-AmpB nanoparticles}

All nanoparticles used in this study were prepared according to the PEC technique. ${ }^{22}$ To prepare the NQ nanoparticles, Cs (5 mg, MW 60,000-120,000 g/mol, [Sigma-Aldrich, St Louis, MO, USA]) was dissolved in $2 \%(\mathrm{v} / \mathrm{v})$ acetic acid in a sodium triphosphate (TPP) buffer $(1 \mathrm{mg} / \mathrm{mL}$; SigmaAldrich), under magnetic stirring (Color Squid IKAMAG ${ }^{\circledR}$ White, IKA Works, Inc., Wilmington, NC, USA) at room temperature. The mixture was sonicated (Ultra Cleaner 1400A, Unique, São Paulo, Brazil) for 30 minutes and the 
$\mathrm{pH}$ adjusted to 6.0-6.5. The nanoparticles were purified using Amicon ${ }^{\circledR}$ Ultra-15 filters with PLHK Ultracel-PL Membrane (100 kDa; Millipore Corporation, MA, USA), followed by centrifugation at $9,000 \times g$ for 20 minutes at $20^{\circ} \mathrm{C} .{ }^{34,35}$

To prepare the NQC nanoparticles, $5 \mathrm{mg}$ of Cs was dissolved in $2 \mathrm{~mL}$ of $2 \%$ acetic acid (v/v). ChS (MW 50,000 g/mol; Henrifarma, São Paulo, Brazil) was dissolved in purified water to obtain solutions with concentrations $2.5,5.0,10.0,25.0$, and $50 \mathrm{mg} / \mathrm{mL}(\mathrm{w} / \mathrm{v})$; with the $\mathrm{Cs} / \mathrm{ChS}$ solutions reaching theoretical ratios of $1: 1,1: 2,1: 5,1: 10$, and 1:20 (w/w), respectively, at $\mathrm{pH} 5.0$. The nanoparticles were spontaneously formed by incorporating $2 \mathrm{~mL}$ of the different concentrations of $\mathrm{ChS}$, as described, and $1 \mathrm{~mL}$ of TPP buffer in purified water $(1 \mathrm{mg} / \mathrm{mL})$ before sonicating for 30 minutes ( $\mathrm{pH}$ 6.0-6.5). Next, the nanoparticles were purified using Amicon filters, followed by centrifugation at $9,000 \times \mathrm{g}$ for 20 minutes at $20^{\circ} \mathrm{C} .^{22}$

To prepare the NQC-AmpB nanoparticles, AmpB (586 $\mu \mathrm{g} / \mathrm{mL}$; Cristália Produtos Químicos Farmacêuticos Ltda, São Paulo, Brazil) was dissolved in 2\% acetic acid (v/v), pH 2.0, followed by the incorporation of Cs into this solution. The subsequent methodology was the same as that used to prepare the NQC nanoparticles.

\section{Physicochemical characterization of nanoparticles \\ Photon correlation spectroscopy (PCS) and zeta potential analysis}

This analytical procedure allows the determination of the mean lengths of the nanoparticles (NQ, NQC, NQC-AmpB) and of the polydispersity index (PI), which is a dimension measure of the broadness of the particle-size distribution. A Zetasizer Nano Zs (Malvern Instruments Ltd, Malvern, UK) apparatus was used for this purpose, after adequate dilution in ultra-pure Milli-Q water. The zeta potential was determined by laser Doppler anemometry using the same equipment. The samples were analyzed following a 1:1,000 dilution in $1 \mathrm{mM}$ of $\mathrm{NaCl}$, and a conductivity of approximately $120 \pm 20$ $\mathrm{S} / \mathrm{cm}^{2}$ to maintain a constant ionic strength.

\section{Scanning electron microscopy (SEM)}

and transmission electron microscopy (TEM)

The size and morphology of the nanoparticles were viewed using SEM (FEI Quanta 200 F, FEI Company, Hillsboro, OR, USA), and TEM (Tecnai G2-12 Spirit BioTwin, FEI Company). SEM samples were mounted on a carbon adhesive tab and sputter coated (10-15 nm) with gold palladium (60/40 alloy). The images were examined using SEM at $15 \mathrm{kV}$. TEM samples were placed on a carbon-coated
200 mesh copper specimen grid (Agar Scientific Ltd, Essex, UK). In this case, one drop of nanoparticle solution was deposited on the grid, and stained with $2 \%$ phosphotungstic acid using the glow-discharged technique for 1.5 minutes. The grids were allowed to dry for 2 days at room temperature. The images were examined using a TEM at $120 \mathrm{kV}^{22,34,36}$

\section{Determination of drug entrapment efficiency and AmpB loading}

The encapsulation efficiency $(\% \mathrm{EE})$ of $\mathrm{AmpB}$ was evaluated by determining the difference between the concentration of AmpB before preparing the nanoparticles $\left(\mathrm{AmpB}_{\mathrm{t}}\right)$ and the non-incorporated $\mathrm{AmpB}\left(\mathrm{AmpB}_{\mathrm{u}}\right)$. The $\mathrm{AmpB}$ that remained insoluble was removed from the formulation by centrifugation. The precipitate was diluted in a methanol:dimethyl sulfoxide (DMSO) solution $(9: 1, \mathrm{v} / \mathrm{v})$ to determine the concentration of non-incorporated AmpB. The influence of the drug concentration on the external aqueous phase of the formulations was considered non-significant due to the poor aqueous solubility of AmpB. ${ }^{37,38}$ The amount of AmpB was measured by ultraviolet-visible (UV-Vis) spectroscopy after adequate dilution. The $\mathrm{EE} \%$ and loading of $\mathrm{AmpB}$ were calculated by the following equations:

$$
\mathrm{EE}(\%)=\frac{\text { Mass of } \mathrm{AmpB}_{\mathrm{t}}-\mathrm{mass}_{\mathrm{s}} \mathrm{AmpB}_{\mathrm{u}}}{\text { mass of } \mathrm{AmpB}_{\mathrm{t}}} \times 100
$$

AmpB loading

$$
(\% \mathrm{w} / \mathrm{w})=\frac{\text { Mass of } \mathrm{AmpB}_{\mathrm{t}}-\text { mass of } \mathrm{AmpB}_{\mathrm{u}}}{\text { Mass of polymers }} \times 100
$$

\section{UV-Vis spectrophotometric assay}

The concentration of $\mathrm{AmpB}$ and the release studies were determined by UV-Vis spectrophotometric assay. ${ }^{39}$ The values of absorbances were evaluated at $\lambda 406 \mathrm{~nm}$ (UV 160 UV-Vis Spectrophotometer, Shimadzu Corporation, Kyoto, Japan) using a quartz cuvette. Two calibration curves were obtained - the first for the concentration of AmpB in the formulations, which was determined using a methanol:DMSO solution $(9: 1, \mathrm{v} / \mathrm{v})$, and the second to evaluate AmpB in the release studies, which was performed using a methanol:phosphate-buffered saline (PBS) solution $(40: 60 \% \mathrm{v} / \mathrm{v}), \mathrm{pH} 7.4$, both with a linearity ranging from 5 to $50 \mu \mathrm{g} / \mathrm{mL}$.

The first analytical curve for AmpB performed to study the $\% \mathrm{EE}$ and $\mathrm{AmpB}$ loading presented a regression equation of $y=0.093 x+0.0585$ and linearity $\left(r^{2}\right)$ of 0.9951 . The analytical curve of AmpB prepared in methanol:PBS 
to evaluate the release presented a regression equation of $\mathrm{y}=0.0909 \mathrm{x}+0.0835$, and linearity $\left(\mathrm{r}^{2}\right)$ of 0.9951 .

\section{Release of AmpB}

The release of $A m p B$ from the NQC-AmpB formulation and an AmpB solution was studied by the dialysis method, while the concentration of the product was estimated by the UVVis method. Briefly, $100 \mu \mathrm{L}$ of NQC-AmpB nanoparticles, equivalent to $1 \mathrm{mg}$ of $\mathrm{AmpB}$, was resuspended in $2 \mathrm{~mL}$ of methanol/DMSO $(9: 1, \mathrm{v} / \mathrm{v})$ in a dialysis bag (MWCO 1,052 Da; Sigma-Aldrich), and dialyzed in $100 \mathrm{~mL}$ of PBS:methanol (60:40\%, v/v). The mixture was continuously stirred at room temperature with a small magnetic bar to ensure homogeneity. Samples ( $1 \mathrm{~mL}$ ) were collected at 1 hour and 2, 3, 4, 6, 8, 12, 24, and 48 hours after, and washed with a fresh solution of PBS:methanol $(60: 40 \%, v / v) .39,40$

\section{UV-Vis spectral study}

UV-Vis spectra of pure AmpB and the NQC-AmpB formulation were obtained using a UV-Vis spectrophotometer. Briefly, the NQC-AmpB formulation was dispersed in deionized water to reach a $A m p B$ concentration of $10 \mu \mathrm{g} / \mathrm{mL}$, then it was scanned in the range $300-500 \mathrm{~nm}$ using plain nanoparticles as blank, and compared with the spectrum of a solution containing AmpB $(10 \mu \mathrm{g} / \mathrm{mL})$, diluted in methanol.

\section{Biological activity of nanoparticles}

\section{Parasites and mice}

L. amazonensis (strain IFLA/BR/1967/PH-8) and L. chagasi (strain MHOM/BR/1970/BH46) were used in this study. The parasites were grown at $24^{\circ} \mathrm{C}$ in Schneider's medium (Sigma-Aldrich), supplemented with $10 \%$ heat-inactivated fetal bovine serum (Sigma-Aldrich), $20 \mathrm{mM} \mathrm{L-glutamine,}$ $200 \mathrm{U} / \mathrm{mL}$ penicillin, and $100 \mu \mathrm{g} / \mathrm{mL}$ streptomycin, $\mathrm{pH}$ 7.4. ${ }^{41}$ Murine macrophages were collected from the peritoneal cavities of female BALB/c mice (8 weeks old) purchased of Institute of Biological Sciences (ICB) from Federal University of Minas Gerais (UFMG). The UFMG Animal Use Committee approved the experimental protocol (code number 182/2012).

\section{Anti-leishmanial activity and cytotoxicity evaluation}

The anti-leishmanial activity was assessed using stationary promastigotes of L. amazonensis and L. chagasi ( 1 x 106 of each species) in the presence of individual concentrations $(0.8-100.0 \mu \mathrm{g} / \mathrm{mL})$ of $\mathrm{Cs}$; $\mathrm{ChS}$; and NQ, NQC, and NQC-AmpB nanoparticles in 96-well culture plates (Corning Life Sciences, Corning, NY, USA), for 48 hours at $24^{\circ} \mathrm{C}$. Pure
AmpB $(0.1-10.0 \mu \mathrm{g} / \mathrm{mL})$ was used as a positive control. The cytotoxicity was assessed by cultivating murine macrophages $\left(1 \times 10^{6}\right.$ cells $)$ in the presence of individual concentrations $(0.8-100.0 \mu \mathrm{g} / \mathrm{mL})$ of $\mathrm{Cs}$; $\mathrm{ChS}$; and NQ, NQC, and NQCAmpB nanoparticles in 96-well culture plates (Corning Life Sciences), for 48 hours at $24^{\circ} \mathrm{C}$. AmpB $(0.1-10.0 \mu \mathrm{g} / \mathrm{mL})$ was also used as a toxicity control. Cell viability was assessed by measuring the cleavage of $2 \mathrm{mg} / \mathrm{mL}$ of 3-(4.5-dimeth ylthiazol-2-yl)-2.5-diphenyl tetrazolium bromide (MTT; Sigma-Aldrich). Absorbances were measured by using a multi-well scanning spectrophotometer (LAB-660, Labtrade of Brazil, São Paulo, Brazil), at a wavelength of $570 \mathrm{~nm}$. Results are expressed as the mean percentage reduction of parasites or macrophages compared with non-treated control wells, and are represented by $50 \%$ inhibitory concentration for Leishmania $\left(\mathrm{IC}_{50}\right)$ and macrophages $\left(\mathrm{CC}_{50}\right) \cdot{ }^{42} \mathrm{In}$ addition, the selectivity index was calculated by determining the ratio between the $\mathrm{CC}_{50}$ and $\mathrm{IC}_{50}$ values.

\section{Hemolytic activity}

The hemolytic activity was investigated by incubating the products $(0.8-100.0 \mu \mathrm{g} / \mathrm{mL})$ with a $5 \% \mathrm{O}^{+}$human red blood cell suspension for 1 hour at $37^{\circ} \mathrm{C}$. AmpB $(1-100 \mu \mathrm{g} / \mathrm{mL})$ was used as a control. The erythrocyte suspension was centrifuged $(1,000 \times g$ for 5 minutes), and cell lysis was determined spectrophotometrically $(540 \mathrm{~nm})$, as described. ${ }^{43}$ The absence (negative control) or presence of hemolysis (positive control) was determined by replacing the tested substance with an equal volume of PBS or distilled water, respectively. The results were determined by the percentage of hemolysis compared with the results obtained using the negative and positive controls. ${ }^{44}$ The results were expressed as the mean percentage reduction in $\mathrm{O}^{+}$human red blood cells compared with non-treated control wells, and represented by the $50 \%$ hemolytic concentration $\left(\mathrm{RBC}_{50}\right)$.

\section{Treatment of infected macrophages}

Murine macrophages $\left(5 \times 10^{5}\right.$ cells $)$ were plated on round glass coverslips inside the wells of a 24-well culture plate (Nunc ${ }^{\mathrm{TM}}$ Culture-Treated Multidish, Thermo Fisher Scientific, Waltham, MA, USA) in a Roswell Park Memorial Institute (RPMI) 1640 medium supplemented with $20 \%$ fetal bovine serum, $2 \mathrm{mM}$ L-glutamine, $200 \mathrm{U} / \mathrm{mL}$ penicillin, and $100 \mu \mathrm{g} / \mathrm{mL}$ streptomycin, at $\mathrm{pH} 7.4$. After 24 hours of incubation at $37^{\circ} \mathrm{C}$ in $5 \% \mathrm{CO}_{2}$, stationary promastigotes of L. amazonensis were added to the wells $\left(5 \times 10^{6}\right.$ cells $)$, and the cultures were incubated for 24 hours at $37^{\circ} \mathrm{C}$ in $5 \% \mathrm{CO}_{2}$. A titration curve was performed prior to this to determine 
the minimum incubation time required for the parasites to infect the macrophages to the maximum level of infection (data not shown). Next, free parasites were removed by extensive washing with RPMI 1640 medium, and the infected macrophages were quantified and treated by 48 hours with either pure Cs and ChS, as well as NQ,... NQ NQC (at 25, 50 , and $100 \mu \mathrm{g} / \mathrm{mL}$, each), or NQC-AmpB nanoparticles or AmpB $(0.04,0.08$, and $0.80 \mu \mathrm{g} / \mathrm{mL}$, each), for 48 hours at $24^{\circ} \mathrm{C}$ in $5 \% \mathrm{CO}_{2}$. Then, cells were washed in RPMI 1640 and incubated with 4\% paraformaldehyde for 15 minutes, after which time they were treated with $70 \%$ ethanol in an ice-bath for 4 hours, before again being washed three times with sterile PBS. The inhibition of Leishmania intra-macrophage viability (in percentage) was determined by counting 200 cells in triplicate. ${ }^{45}$ Results shown in this study are representative of three independent experiments, performed in triplicate, which presented similar results.

\section{Intracellular localization of the nanoparticles Preparation of FITC-labeled nanoparticles}

The nanoparticles were labeled with FITC fluorophore (Sigma-Aldrich) to perform fluorescence microscopy. First, Cs was conjugated with the fluorophore, and this was followed by the preparation of different nanoparticles. The FITC-labeled Cs was based on the reaction between the isothiocyanate group of FITC and the primary amino group of Cs. ${ }^{46}$ Dehydrated methanol $(100 \mathrm{~mL})$ containing $2 \mathrm{mg} / \mathrm{mL}$ of FITC was added to $1 \%(\mathrm{w} / \mathrm{v}) \mathrm{Cs} / \mathrm{hydrochloride}$ in $0.1 \mathrm{M}$ acetic acid. After 3 hours of incubation at room temperature and in the dark, the FITC-labeled Cs was precipitated in $0.2 \mathrm{M} \mathrm{NaOH}$ and separated from non-reacted FITC by ultrafiltration at $10,000 \times g$ for 30 minutes, after which time the product was decanted using a freeze-drying system. ${ }^{47}$ The nanoparticles were used immediately upon preparation.

\section{Co-localization studies of macrophages infected with L. chagasi pX63NEO-mCherry and treated with FITC-labeled nanoparticles}

To analyze the intracellular distribution and the biological activity of the NQ, NQC, and NQC-AmpB nanoparticles into the macrophages, L. chagasi episomal pX63NEO-mCherry strain (MHOM/BR/BA262) was used. This strain, which naturally presents a red color when evaluated by fluorescence, was kindly provided by Dr Manoel Barral Netto (Fiocruz, Salvador, Brazil).
Macrophages $\left(5 \times 10^{5}\right)$ were plated in a 24-well microplate with sterile coverslip glass and infected with parasites (1:10 ratio) for 4 hours, washed twice, and incubated for 24 hours at $24^{\circ} \mathrm{C}$. After incubation, infected cells were incubated with FITC-conjugated nanoparticles (FITC-NQ, FITC-NQC, and FITC-NQC-AmpB) at a concentration of $5 \mu \mathrm{g} / \mathrm{mL}$ for 2 hours at $37^{\circ} \mathrm{C}$ in $5 \% \mathrm{CO}_{2}$. To determine the fluorescence background of the nanoparticles, non-labeled nanoparticles (NQ, NQC, and NQC-AmpB) were used as controls. To confirm that the infected cells were in fact macrophages, these were incubated with a monoclonal anti-F4/80-Pecy. 5 antibody for 20 minutes at $37^{\circ} \mathrm{C}$ in $5 \% \mathrm{CO}_{2}$. The fluorescence parameters used were: FITC: $\lambda$ exc $485 \mathrm{~nm}$ and $\lambda$ emi 535 nm; mCherry: $\lambda$ exc $560 \mathrm{~nm} \lambda$ emi $635 \mathrm{~nm}$; anti-F4/80-Pecy. 5 antibody: $\lambda$ exc $485 \mathrm{~nm}$ and $\lambda$ emi $780 \mathrm{~nm}$. The overlay of the FITC-labeled nanoparticles and L. infantum chagasimCherry (Lic-mCherry) cells was determined by the presence of yellow/orange fluorescence. All images were taken with a confocal laser-scanning microscope (Zeiss Axiovert $200 \mathrm{M}$, Carl Zeiss, Oberkochen, Germany) equipped with imaging software (LSM 5 Image Browser, Carl Zeiss). ${ }^{47}$

\section{Statistical analyses}

Statistical analyses were performed using GraphPad Prism ${ }^{\mathrm{TM}}$ (version 5.0; GraphPad Software, Inc., La Jolla, CA, USA). Results are representative of three independent experiments, performed in triplicate, which presented similar values. UV-Vis spectrophotometric assay was determined by linear regression and the $r^{2}$ value indicates the measure of goodness-of-fit for linear regression. The Pearson's normality test was used to determine the normality of the data. Since the results were found to be normally distributed, one-way analysis of variance, followed by Tukey's test was also used. Differences were considered significant when $P<0.05$. The $\mathrm{IC}_{50}, \mathrm{CC}_{50}$, and $\mathrm{RBC}_{50}$ curves were determined by applying the sigmoidal regression of the logarithm dose-response results.

\section{Results \\ Physicochemical characterization of prepared nanoparticles}

Figure 1 summarizes the preparation of the nanoparticles. The optimized NQ nanoparticles were obtained with a size of $79 \pm 1 \mathrm{~nm}$ and a PI of $<0.2$ (Figure 2 and Table 1 ). The observation and light scattering results showed that the $\mathrm{pH}$ effect was important. It could be observed that the formation of $\mathrm{Cs}$ and $\mathrm{ChS}$ nanoparticles occurred when the $\mathrm{pH}$ ranged between 4.0 and 5.0. 

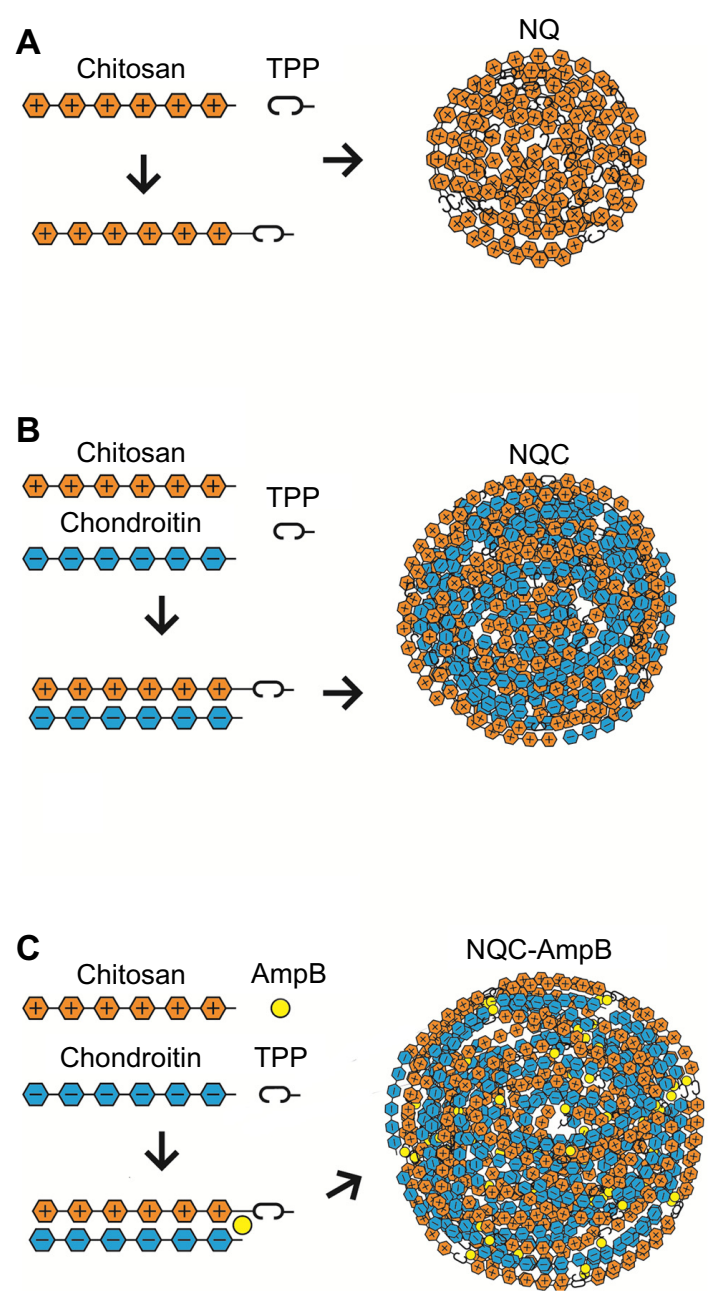

Figure I Preparation of nanoparticles. Diagram demonstrating the modeling of the nanoparticles: (A) NQ, formulated by PEC using Cs and TPP; (B) NQC, formulated by PEC using Cs, ChS, and TPP; and (C) NQC-AmpB, formulated by PEC using Cs, ChS, TPP, and AmpB.

Abbreviations: AmpB, amphotericin B; Cs, chitosan; ChS, chondroitin sulfate; NQ, chitosan nanoparticle; NQC, chitosan-chondroitin sulfate nanoparticle; NQC-AmpB, amphotericin B-chitosan-chondroitin sulfate nanoparticle; PEC, polyelectrolyte complexation; TPP, sodium triphosphate.

The effect of the polymer ratio on the production of NQC nanoparticles was studied using a fixed amount of Cs. The size of NQC and NQC-AmpB nanoparticles depended on the ratio between $\mathrm{Cs}$ and $\mathrm{ChS}$ of each formulation (Figure 2 and Table 1). The NQC nanoparticles ranged in size from $104 \pm 3 \mathrm{~nm}(1: 1)$ to $135 \pm 9 \mathrm{~nm}(1: 20)$. When the AmpB was added, the nanoparticle size was increased (NQC-AmpB 1:1, $136 \pm 11 \mathrm{~nm})$. All nanoparticles were obtained with a relatively narrow size distribution - that is, PI $\leq 0.2$ - and were analyzed and considered positively charged (range +8.4 to $+30.2 \mathrm{mV}$ ). The morphology of the nanoparticles was examined by SEM and TEM, and the results showed that the individual particles were of uniform spherical shape with a smooth surface, and were uniformly distributed throughout all formed product (Figure 2).

The entrapment efficiency of AmpB ranged between $89 \%$ and $92 \%$, while the loading of this product ranged from $1 \%$ to $11 \%(\mathrm{w} / \mathrm{w}$; Figure $3 \mathrm{~A})$. The release profiles of AmpB through synthetic membrane from different formulations are showed in Figure 3B. The AmpB content in the release medium was evaluated spectrophotometrically. The percentage of AmpB released from NQC-AmpB 1:1, NQC-AmpB 1:2, NQC-AmpB 1:5, NQC-AmpB 1:10, and NQC-AmpB 1:20 nanoparticles after 48 hours in a methanol/DMSO solution $(9: 1, \mathrm{v} / \mathrm{v})$ was $57 \% \pm 6 \%$, $58 \% \pm 3 \%, 61 \% \pm 6 \%, 68 \% \pm 2 \%$, and $59 \% \pm 1 \%$, respectively. Therefore, the AmpB release from solution used as control was significantly higher than that obtained from the other formulations.

The UV spectrum of AmpB diluted in methanol exists like a monomer, presenting four different absorption bands of decreasing intensity at 405, 385, 365, and $344 \mathrm{~nm}$. As aggregation occurs and the self-associated form is also emerged the small band at 344 undergoes a blue shift to about 333, increasing in intensity, and the other three bands undergo shifts to about 368,390 , and $420 \mathrm{~nm}$, respectively, as well as reducing in intensity, indicating the appearance of the self-aggregated form. A comparison of UV spectra of AmpB diluted in methanol and of NQC-AmpB formulation is shown in Figure 3C. The changes in the spectrum clearly indicate the presence of the self-associated form of AmpB in the NQC-AmpB formulation.

Attenuated total reflectance-Fourier transform infrared spectroscopy (ATR-FTIR) was applied to probe chemical reactions or interactions among different substances. Figure 4 shows the ATR-FTIR spectra of Cs, ChS, NQC, NQC-AmpB nanoparticles, and pure AmpB. Using only Cs, two characteristic absorption bands at 1,634 and $1,539 \mathrm{~cm}^{-1}$ were detected, which were attributed to amide $\mathrm{I}(\mathrm{C}=\mathrm{O})$ and amine $(\mathrm{N}-\mathrm{H})$ vibration overlapping the amide II vibration, respectively. The overlapped wide absorption band around $3,267 \mathrm{~cm}^{-1}$ was due to the stretching vibration of the $\mathrm{O}-\mathrm{H}$ bonded to the $\mathrm{N}-\mathrm{H}$ in Cs. The characterization peaks in the ChS spectrum were: $3,356 \mathrm{~cm}^{-1}$, which could be attributed to the $-\mathrm{OH},-\mathrm{NH} 2$, and $-\mathrm{CH}$ stretching; $1,645 \mathrm{~cm}^{-1}$, attributed to the $\mathrm{C}=\mathrm{O}$ of the amide I band stretching; a shoulder in $1,609 \mathrm{~cm}^{-1}$, attributed to $\mathrm{N}-\mathrm{H}$ deformation; a band in $1,315 \mathrm{~cm}^{-1}$, attributed to $\mathrm{S}=\mathrm{O}$ stretching; and a band in $1,062 \mathrm{~cm}^{-1}$, attributed to $\mathrm{C}-\mathrm{SO}_{3}^{-}$deformation. The overlapped wide absorption band around $3,356 \mathrm{~cm}^{-1}$ was 

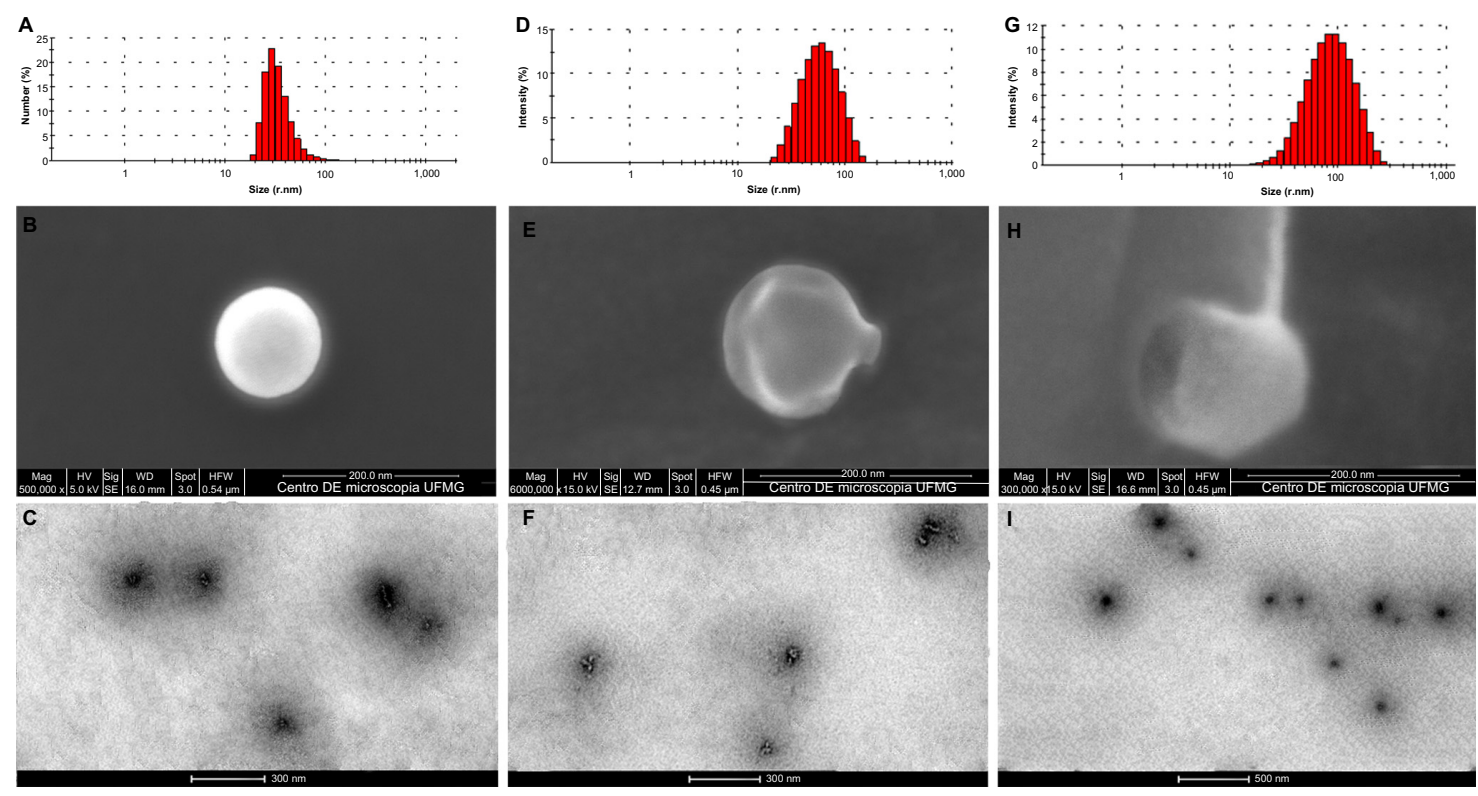

Figure 2 Size, polydispersity index (PI), and morphology of the engineered nanoparticles: (A) particle-size distribution of NQs determined by photon correlation spectroscopy (PCS), (B) scanning electron microscopy (SEM) image of an NQ, (C) transmission electron microscopy (TEM) image of NQs, (D) particle-size distribution of NQCs by PCS, (E) SEM image of an NQC, (F) TEM image of NQCs, (G) particle-size distribution of NQC-AmpB by PCS, (H) SEM image of an NQC-AmpB, and (I) TEM image of NQC-AmpB

Abbreviations: NQ, chitosan nanoparticle; NQC, chitosan-chondroitin sulfate nanoparticle; NQC-AmpB, chitosan-chondroitin sulfate-amphotericin B nanoparticle; TPP, sodium triphosphate.

due to the stretching vibration of the $\mathrm{O}-\mathrm{H}$ bonded to the $\mathrm{N}-\mathrm{H}$ in $\mathrm{ChS}$.

In the NQC nanoparticles, the $1,634 \mathrm{~cm}^{-1}$ peak of the amide I band shifted to $1,640 \mathrm{~cm}^{-1}$, whereas the $1,539 \mathrm{~cm}^{-1}$ peak (N-H deformation) shifted to $1,557 \mathrm{~cm}^{-1}$, and the $1,315 \mathrm{~cm}^{-1}$ peak shifted to $1,262 \mathrm{~cm}^{-1}$ ( $\mathrm{S}=\mathrm{O}$ stretching), due to the electrostatic interactions and hydrogen bonds that probably occurred between these groups, when Cs and ChS were used in the formulation. The intensity and width of the bands of the sulfate group of the $\mathrm{ChS}$ spectra decreased in intensity in NQC and NQC-AmpB spectra, showing the interactions between the two polymers. In the NQC-AmpB spectra, no significant difference could be observed when compared with the NQC spectra.

\section{Biological characterization of the nanoparticles}

The in vitro anti-leishmanial activity of the pure $\mathrm{Cs}$ and $\mathrm{ChS}$, as well as NQ, NQC, and NQC-AmpB nanoparticles was evaluated using the stationary promastigotes of L. amazonensis and L. chagasi. Parasites were incubated with the products for 48 hours at $24^{\circ} \mathrm{C}$. The $\mathrm{Cs}$ and $\mathrm{ChS}$ presented $\mathrm{IC}_{50}$ values of $73 \pm 5$ and $66 \pm 1 \mu \mathrm{g} / \mathrm{mL}$, respectively, for L. amazonensis; and of $67 \pm 1$ and $71 \pm 10 \mu \mathrm{g} / \mathrm{mL}$, respectively, for $L$. chagasi (Table 2 ). The NQ and NQC nanoparticles presented $\mathrm{IC}_{50}$ values of $52 \pm 2$ and
$44 \pm 2 \mu \mathrm{g} / \mathrm{mL}$, respectively, for L. amazonensis; and of $46 \pm 6$ and $39 \pm 1 \mu \mathrm{g} / \mathrm{mL}$, respectively, for $L$. chagasi. In the evaluation of the anti-leishmanial activity of the AmpB loaded in NQC$\mathrm{AmpB}$, the $\mathrm{IC}_{50}$ values were $1 \pm 0$ and $0.1 \pm 0 \mu \mathrm{g} / \mathrm{mL}$ against $L$. amazonensis and L. chagasi, respectively. Using pure AmpB the $\mathrm{IC}_{50}$ values were $0.1 \pm 0$ and $0.1 \pm 0 \mu \mathrm{g} / \mathrm{mL}$ against $L$. amazonensis and L. chagasi, respectively.

The cytotoxicity of $\mathrm{Cs}, \mathrm{ChS}, \mathrm{NQ}, \mathrm{NQC}$, and NQC-AmpB nanoparticles was also investigated. The assays revealed no significant toxicity in mammals' cells using the highest concentration $(100 \mu \mathrm{g} / \mathrm{mL})$ of $\mathrm{Cs}$; $\mathrm{ChS}$; and NQ, NQC, and NQC-AmpB nanoparticles (Table 2). Using pure AmpB, the $\mathrm{CC}_{50}$ was $1 \pm 0 \mu \mathrm{g} / \mathrm{mL}$; however, when $\mathrm{AmpB}$ was loaded in the NQC nanoparticles, a nearly ten-fold decrease in cytotoxicity was observed $(9 \pm 0 \mu \mathrm{g} / \mathrm{mL})$.

To study the safety of the nanoparticles for systemic application in patients, the toxicity of the products was assessed in human $\mathrm{O}^{+}$human red blood cells (Table 2 ). No tested products were found to significantly damage the $\mathrm{O}^{+}$human red blood cells. A RBC $\mathrm{R}_{50}$ of $12 \pm 3 \mu \mathrm{g} / \mathrm{mL}$ was found for $\mathrm{AmpB}$, and of $240 \pm 33 \mu \mathrm{g} / \mathrm{mL}$ for the NQC-AmpB nanoparticles. Thus, this formulation was significantly - nearly ten-fold - less hemolytic than pure AmpB.

An evaluation of the capacity of the Cs; ChS; and NQ, NQC, and NQC-AmpB nanoparticles in treating macrophages 


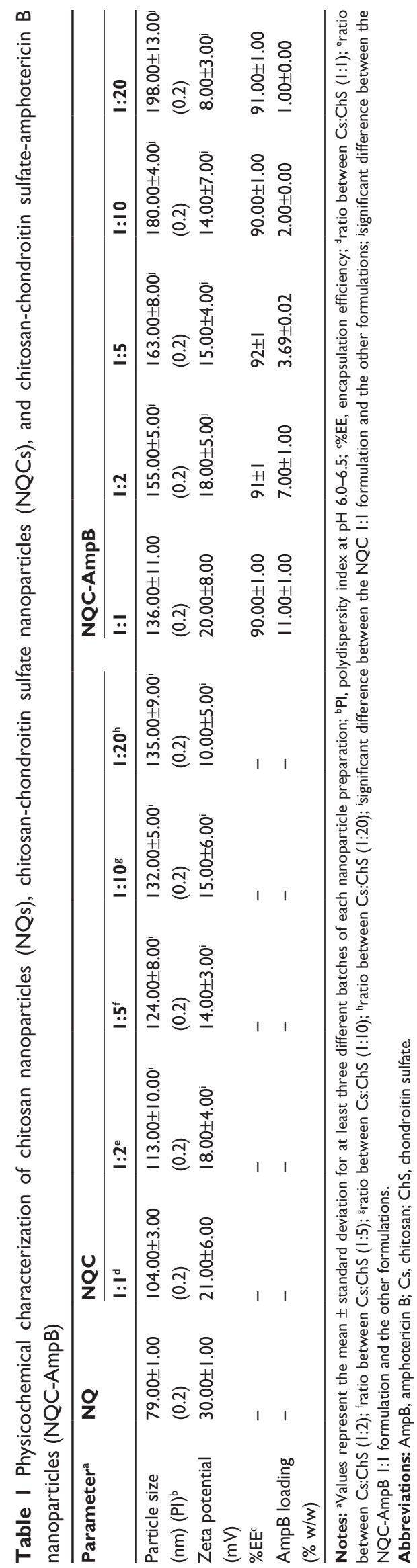

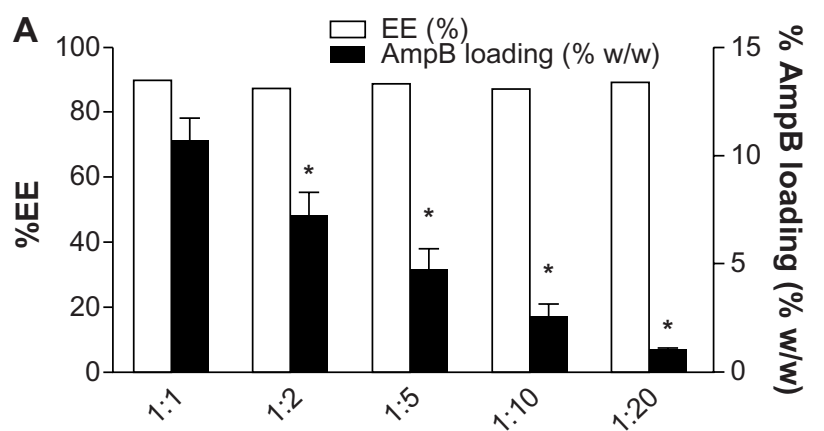

Proportion between chitosan and chondroitin in NQC-AmpB

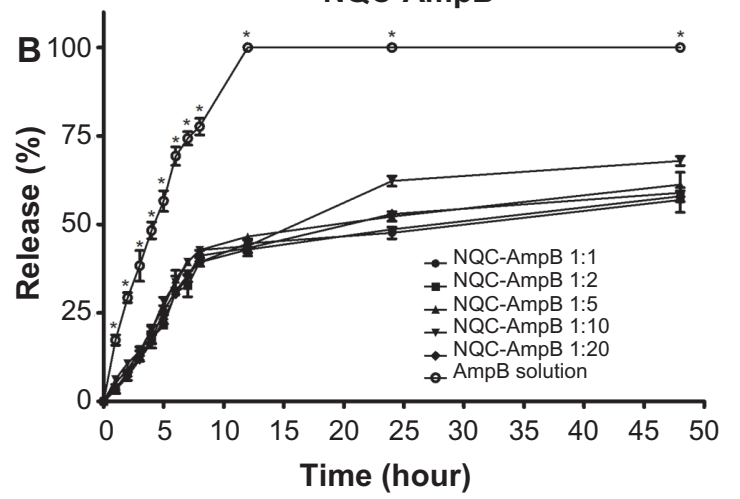

C

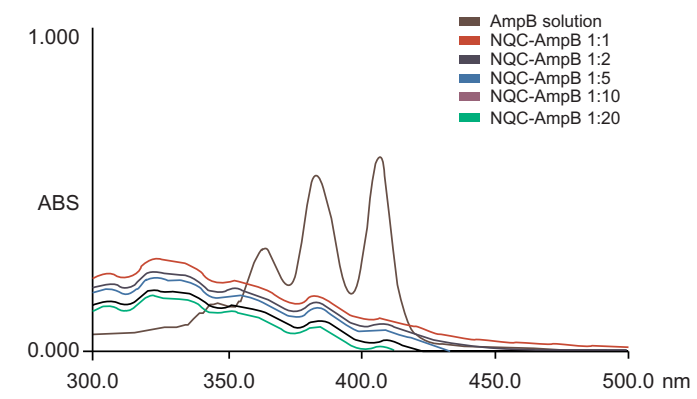

Figure 3 Physicochemical characterization of the engineered nanoparticles. (A) Encapsulation efficiency (\%EE) and drug loading (\% w/w) of different ratios of Cs:ChS into NQC-AmpB. Bars indicate the mean \pm standard deviation of each evaluated formulation ( $n=3$, per group). (B) Release profile of AmpB from NQCAmpB in different ratios of Cs:ChS and AmpB. (C) Study of aggregation state of AmpB/ultraviolet spectrum in a methanol solution, and its loading into the NQCs. One-way analysis of variance followed by Tukey's test were used, and a probability of $5 \%$ was considered significant using GraphPad Prism.

Notes: *Significant difference in a I:I ratio between $\mathrm{Cs}$ and $\mathrm{ChS}$ in the NQCAmpB formulation when compared with the other formulations $(P<0.05)$.

Abbreviations: $\mathrm{AmpB}$, amphotericin $\mathrm{B}$; $\mathrm{Cs}$, chitosan; $\mathrm{ChS}$, chondroitin sulfate; $\mathrm{NQ}$, chitosan nanoparticle; NQC, chitosan-chondroitin sulfate nanoparticle; NQCAmpB, chitosan-chondroitin sulfate-amphotericin B nanoparticle.

infected with $L$. amazonensis was also performed. For this, cells were infected with promastigotes of $L$. amazonensis (10 parasites per 1 macrophage), and later treated with 25, 50, and $100 \mu \mathrm{g} / \mathrm{mL}$ of Cs; ChS; NQ or NQC nanoparticles, or with $0.04,0.08$, and $0.8 \mu \mathrm{g} / \mathrm{mL}$ of NQC-AmpB nanoparticles or pure $\mathrm{AmpB}$, for 48 hours at $24^{\circ} \mathrm{C}$ and $5 \% \mathrm{CO}_{2}$. The results showed that infected and non-treated macrophages presented a degree of infection of $87 \% \pm 5 \%$, and an average number of 


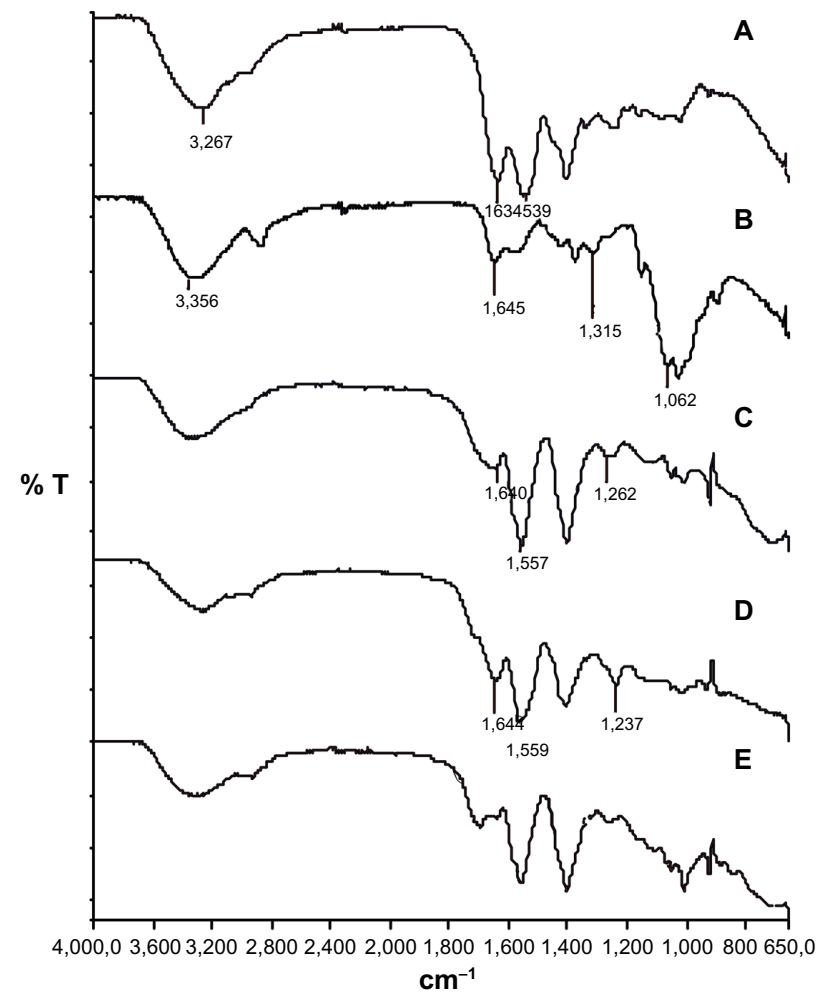

Figure 4 Attenuated total reflectance-Fourier-transform infrared spectroscopy spectra of $(\mathbf{A})$ chitosan, (B) chondroitin sulfate, $(\mathbf{C})$ chitosan-chondroitin sulfate nanoparticles, (D) chitosan-chondroitin sulfate-amphotericin B nanoparticles, and (E) pure amphotericin B.

amastigotes per macrophage of $8 \pm 1$. When mammals' cells were treated with Cs; ChS; and NQ and NQC nanoparticles $(100 \mu \mathrm{g} / \mathrm{mL}$, each), the degree of infection was $66 \% \pm 6 \%$, $60 \% \pm 4 \%, 39 \% \pm 2 \%$, and $29 \% \pm 2 \%$, respectively, and the average number of amastigotes per macrophage was $3.7 \pm 0.3$, $3.1 \pm 0.2,2.1 \pm 0.3$, and $2 \pm 0.2$, respectively. Using NQC-AmpB nanoparticle or pure AmpB to treat infected cells $(0.8 \mu \mathrm{g} / \mathrm{mL}$, each), the degree of infection was $8 \% \pm 0.5 \%$ and $9 \% \pm 1 \%$, respectively; and the average number of amastigotes per macrophage was $0.2 \pm 0.1$ and $0.3 \pm 0.1$, respectively. In this context, macrophages infected and later treated with pure Cs and ChS, as well as NQ, NQC, or NQC-AmpB nanoparticles; or pure $\mathrm{AmpB}$ presented reductions in the parasite load in the order of $24 \%, 31 \%, 55 \%, 66 \%, 90 \%$, and $89 \%$, respectively (Table 3 ).

To determine the intracellular location of the nanoparticles in the infected and treated macrophages, confocal microscopy was performed. In the results, it was possible to observe a cytoplasmatic localization of the tested NQ, NQC, and NQC-AmpB formulations (Figure 5). Macrophages infected with $\mathrm{pX} 63$ NEO-mCherry $L$. chagasi were incubated with the FITC-labeled nanoparticles, to analyze whether the intracellular allocation of these formulations could, in fact, overlap with the parasite localization. All the formulations showed intracellular co-localization with the parasites, indicating that these products could indeed reach the parasitophorous vacuoles. In addition, non-viable parasites were also visualized in the experiments, indicating the anti-leishmanial efficacy of the evaluated nanoparticles.

\section{Discussion}

The current treatments for leishmaniasis have been considered unsatisfactory mainly due to the high toxicity of the products and the growing resistance of the parasites to the drugs. ${ }^{49}$ In this context, the purpose of the study reported here was to find new formulations based on engineering nanoparticles to carry a known drug to treat leishmaniasis, AmpB. This product is an effective anti-leishmanial agent, but is highly toxic to mammals' cells. In this study, AmpB was associated with two anti-leishmanial compounds, $\mathrm{ChS}$, a substance described here for the first time as having

Table 2 Anti-leishmanial activity $\left(\mathrm{IC}_{50}\right)$, cytotoxicity $\left(\mathrm{CC}_{50}\right)$, hemolytic activity $\left(\mathrm{RBC}_{50}\right)$, and selectivity index (SI) of chitosan (Cs), chondroitin (ChS), chitosan nanoparticles (NQs), chitosan-chondrotin nanoparticles (NQCs), amphotericin B-chitosan-chondroitin nanoparticles (NQC-AmpB), and pure amphotericin B (AmpB)

\begin{tabular}{|c|c|c|c|c|c|c|}
\hline \multirow{2}{*}{$\begin{array}{l}\text { Compound/ } \\
\text { formulation }\end{array}$} & \multicolumn{6}{|l|}{ Biological activity } \\
\hline & $\begin{array}{l}\text { Leishmania } \\
\text { amazonensis }\left(\mathrm{IC}_{50}{ }^{\mathrm{e}}\right)\end{array}$ & $\begin{array}{l}\text { Leishmania } \\
\text { chagasi }\left(\mathrm{IC}_{50}\right)\end{array}$ & $\begin{array}{l}\text { Murine macrophages } \\
\left(\mathrm{CC}_{50}{ }^{\circ}\right)\end{array}$ & $\begin{array}{l}\mathrm{SI}^{\mathrm{h}} \\
\text { L. amazonensis }\end{array}$ & $\begin{array}{l}\text { SI } \\
\text { L. chagasi }\end{array}$ & $\begin{array}{l}\text { Red blood cells } \\
\left(\operatorname{RBC}_{50}^{\mathrm{g}}\right)\end{array}$ \\
\hline Cs & $73 \pm 5^{d}$ & $67 \pm 1^{d}$ & $1,559 \pm 10^{d}$ & 22 & 23 & $432 \pm 2 I^{d}$ \\
\hline ChS & $66 \pm 1^{d}$ & $71 \pm 10^{d}$ & $1,349 \pm 142^{d}$ & 20 & 19 & $697 \pm 106^{d}$ \\
\hline $\mathrm{NQ}$ & $52 \pm 2^{\mathrm{a}, \mathrm{d}}$ & $46 \pm 6^{a, d}$ & $831 \pm 13^{a, d}$ & 16 & 18 & $238 \pm 26^{a, d}$ \\
\hline NQC & $44 \pm 2^{b, c, d}$ & $39 \pm I^{b, c, d}$ & $1,189 \pm 67^{b, d}$ & 27 & 31 & $317 \pm 4^{b, d}$ \\
\hline NQC-AmpB & $\mathrm{I} \pm 0$ & $0.1 \pm 0.1$ & $9 \pm 0^{d}$ & 99 & 81 & $240 \pm 33^{d}$ \\
\hline AmpB & $0.1 \pm 0$ & $0.1 \pm 0$ & $\mathrm{I} \pm 0.2$ & 8 & 9 & $12 \pm 3^{d}$ \\
\hline
\end{tabular}

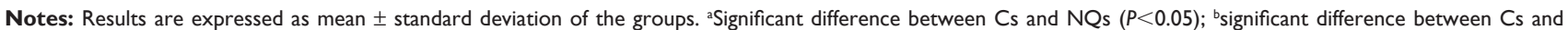
NQCs $(P<0.05)$; 'significant difference between ChS and NQC $(P<0.05)$; dsignificant difference between AmpB and other compounds $(P<0.05)$; elC ${ }_{50}$, concentration needed to inhibit $50 \%$ of the parasites' viability; ${ }^{\mathrm{C}} \mathrm{CC}_{50}$, concentration needed to inhibit $50 \%$ of the macrophages' viability; ${ }^{8 R B C}{ }_{50}$, concentration needed to lysis $50 \%$ of the $\mathrm{O}^{+}$human

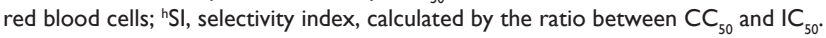


Table 3 Percentage of infected macrophages and parasite burden after treatment with the evaluated formulations

\begin{tabular}{|c|c|c|c|c|}
\hline $\begin{array}{l}\text { Compound/ } \\
\text { formulation }\end{array}$ & $\begin{array}{l}\text { Concentration } \\
(\mu \mathrm{g} / \mathrm{mL})\end{array}$ & $\begin{array}{l}\text { Percentage of infected } \\
\text { macrophages after treatment }\end{array}$ & $\begin{array}{l}\text { Number of amastigotes per } \\
\text { macrophage after treatment }\end{array}$ & $\begin{array}{l}\text { Reduction of internalized } \\
\text { parasites (\%) }\end{array}$ \\
\hline \multirow[t]{3}{*}{ Cs } & 100.00 & $66 \pm 6$ & $4 \pm 0$ & 24 \\
\hline & 50.00 & $68 \pm 6$ & $4 \pm 1$ & 21 \\
\hline & 25.00 & $72 \pm 5$ & $4 \pm 1$ & 17 \\
\hline \multirow[t]{3}{*}{ ChS } & 100.00 & $60 \pm 4$ & $3 \pm 0$ & 31 \\
\hline & 50.00 & $62 \pm 2$ & $3 \pm 1$ & 29 \\
\hline & 25.00 & $70 \pm 5$ & $4 \pm 1$ & 20 \\
\hline \multirow[t]{3}{*}{ NQ } & 100.00 & $39 \pm 2$ & $2 \pm 0$ & 55 \\
\hline & 50.00 & $53 \pm 4$ & $3 \pm 1$ & 29 \\
\hline & 25.00 & $70 \pm 1$ & $4 \pm 2$ & 19 \\
\hline \multirow[t]{3}{*}{ NQC } & 100.00 & $29 \pm 2$ & $2 \pm 0$ & 66 \\
\hline & 50.00 & $44 \pm 3$ & $3 \pm 1$ & 49 \\
\hline & 25.00 & $67 \pm 3$ & $4 \pm 1$ & 23 \\
\hline \multirow[t]{3}{*}{ NQC-AmpB } & 0.80 & $9 \pm 1$ & 0 & 90 \\
\hline & 0.08 & $29 \pm 3$ & $2 \pm 1$ & 66 \\
\hline & 0.04 & $44 \pm 7$ & $3 \pm 1$ & 49 \\
\hline \multirow[t]{3}{*}{ AmpB } & 0.80 & $9 \pm 1$ & 0 & 89 \\
\hline & 0.08 & $34 \pm 5$ & $2 \pm 1$ & 61 \\
\hline & 0.04 & $58 \pm 9$ & $3 \pm 1$ & 34 \\
\hline
\end{tabular}

Notes: Macrophages were plated on round glass coverslips in 24-well culture plates and infected with promastigotes of Leishmania amazonensis (I0 parasites per I macrophage). Free parasites were removed by extensive washing, and infected macrophages were treated with 25,50 , and I00 $\mu \mathrm{g} / \mathrm{mL}$ of Cs, ChS, NQ, NQC, or with 0.04 , 0.08 , and $0.8 \mu \mathrm{g} / \mathrm{mL}$ of $\mathrm{NQC}-\mathrm{AmpB}$ and pure $\mathrm{AmpB}$, for 48 hours at $24^{\circ} \mathrm{C}$ and $5 \% \mathrm{CO}_{2}$. The percentage of infected macrophages, the average number of amastigotes per macrophage after treatment, and the average reduction in percentage of internalized parasites were determined by counting 200 cell coverslips, in triplicate, as compared with the non-treated controls. Results are expressed as medium \pm standard deviation of the percentages of the infected macrophages and by reduction of the internalized parasites in the treated cultures.

Abbreviations: AmpB, amphotericin B; Cs, chitosan; ChS, chondroitin; NQ, chitosan nanoparticle; NQC, chitosan-chondroitin nanoparticle; NQC-AmpB, amphotericin B-chitosan-chondroitin nanoparticle.

anti-leishmanial activity, and $\mathrm{Cs}$, which has been previously proven to have an anti-leishmanial effect. ${ }^{50}$ This formulation was physicochemically characterized and assessed by its anti-leishmanial activity against stationary promastigotes of L. amazonensis and L. chagasi. Studies were extended to establish its $\mathrm{IC}_{50}$ value, as well as its effects on the intramacrophage Leishmania, and its cytotoxic effect on murine macrophages and $\mathrm{O}^{+}$human red blood cells.

In our study, NQC-AmpB nanoparticles were prepared and a physicochemical characterization was also performed to characterize their stability. In addition, the biological activity was also evaluated and this formulation proved to be a notable targeted drug-delivery system to be used in the treatment of disease. In addition to anti-leishmanial activity, NQC-AmpB showed low toxicity to mammals' cells and good performance in treating infected macrophages.

To compose a PEC by combining $\mathrm{Cs}$ and $\mathrm{ChS}$, both polymers should carry opposite charges, which occur at a $\mathrm{pH}$ range within their own $\mathrm{pKa}$ values. Cs possesses a $\mathrm{pKa}$ of near 6.5 , with a maximum of one charge per residue. ${ }^{26}$ The apparent dissociation constant of $\mathrm{ChS}$ has been estimated to be from 4.4 to $4.5 .^{51}$ Therefore, to formulate an effective nanoparticle combining $\mathrm{Cs}$ and $\mathrm{ChS}$, it is necessary to create an electrostatic interaction between $\mathrm{Cs}$ cations and $\mathrm{ChS}$ anions, in which the positively charged amino groups of $\mathrm{Cs}$ interact with the negatively charged sulfate groups of ChS. Thus, the optimal pH necessary to achieve a stable PEC with the highest yield is a $\mathrm{pH}$ of approximately 5.0, as observed in our study, and also described by Chen et al. ${ }^{52}$ The main advantage of this methodology is that it allows for the production of AmpB-loaded nanoparticles without the use of organic solvents or heating.

The NQ nanoparticles were also prepared by a PEC technique using Cs and TPP. ${ }^{34,35}$ This formulation was prepared to analyze the level of efficacy of Cs against Leishmania spp. and the synergistic biological effect of $\mathrm{Cs}$ and $\mathrm{ChS}$ in a nanoparticle system (NQC). The formulations were optimized and the nanoparticles were prepared to be smaller than 200 $\mathrm{nm}$, of homogeneous size, and have a PI $<0.2$. The addition of the sonication step optimized the size of the formulations $(<200 \mathrm{~nm})$, as described by Yeh et al. ${ }^{22}$ When $\mathrm{ChS}$ and $\mathrm{AmpB}$ were added in our study, there was an increase in particle size, as shown in Figure 2 and Table 1. Yeh et al also hypothesized that there is a linear relationship between the particle size and 

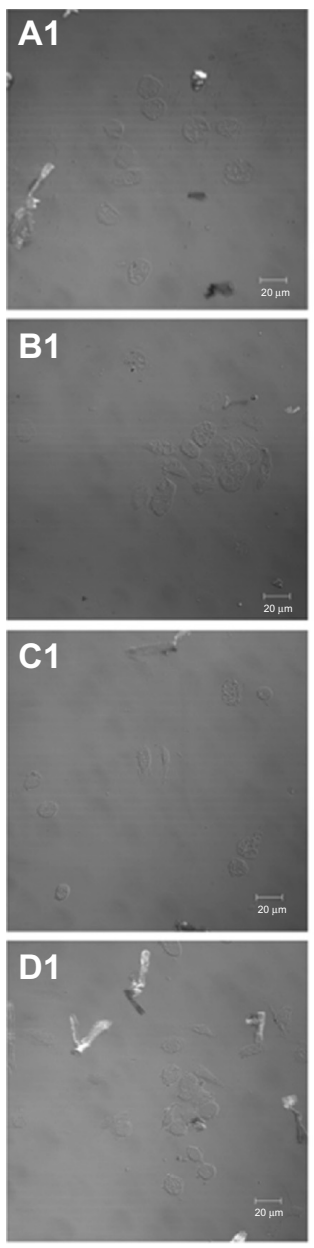
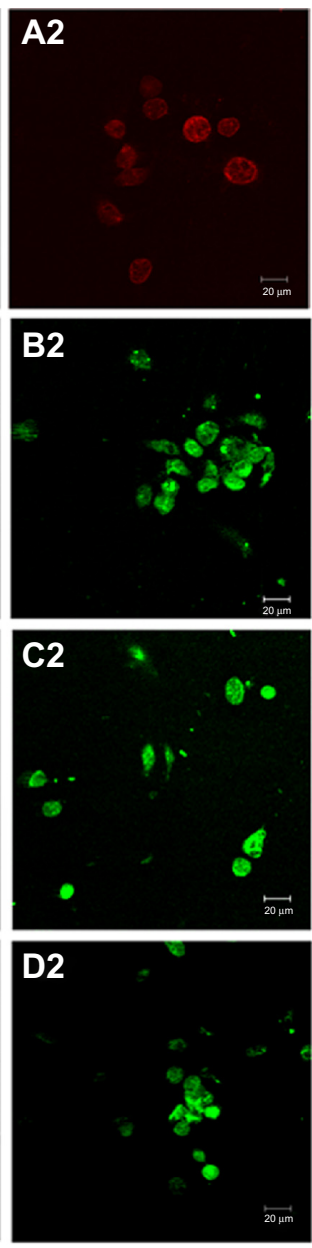
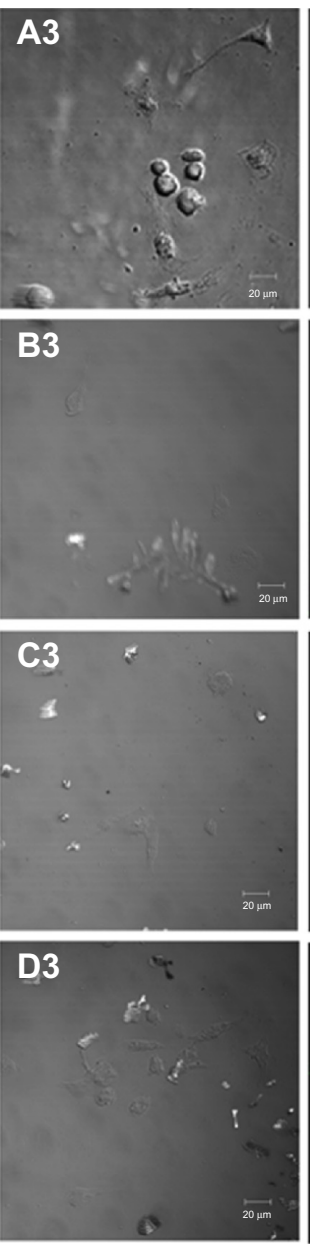
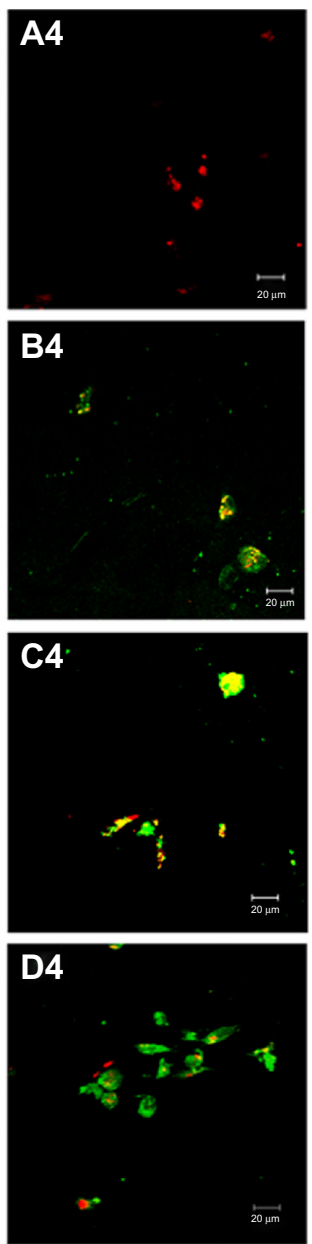

Figure 5 Confocal images of fluorescein isothiocyanate (FITC)-labeled nanoparticles in L. infantum chagasi-mCherry (Lic-mCherry)-infected. (AI and A2) Macrophages (in red, panel A2) after incubation with monoclonal anti-F4/80-Pecy.5 antibody for 20 minutes at $37^{\circ} \mathrm{C}$ in $5 \% \mathrm{CO}_{2}$. Images were obtained using optical and confocal microscopy, respectively. (A3 and A4) Macrophages after infection with Leishmania chagasi pX63NEO-mCherry (in red in A4). Images were obtained using optical and confocal microscopy, respectively. (BI and B2) The presence of FITC-loaded-NQs (green color in B2) accumulated in the cytoplasm of cells. Images were obtained using optical and confocal microscopy, respectively. These characteristics are also shown in CI and C2, and DI and D2, using FITC-loaded-NQCs and FITC-loaded-NQC-AmpB, respectively. (B3 and B4) The FITC-loaded-NQ formulations that could overlap with the parasites (in red). In panels, there is a general overview of macrophages containing nonviable parasites (orange color), which could be to indicate the anti-leishmanial activity of the evaluated products. The same result was observed in C3 and C4, and D3 and D4, where, in both cases, they were exposed to FITC-loaded-NQCs and FITC-loaded-NQC-AmpB, respectively.

Abbreviations: NQ, chitosan nanoparticle; NQC, chitosan-chondroitin sulfate nanoparticle; NQC-AmpB, chitosan-chondroitin sulfate-amphotericin B nanoparticle.

concentration of $\mathrm{ChS}$ in a fixed $\mathrm{ChS} / \mathrm{CS}$ ratio. ${ }^{22}$ Nevertheless, in all cases, the nanoparticles used in this study presented smaller sizes. Cs in $\mathrm{pH} 5.0$ possesses a slightly positive charge, because of its protonation in amino groups, whereas $\mathrm{ChS}$ possesses a negative charge at this $\mathrm{pH}$, because of the sulfate group. As ChS was added to the formulation, a decrease in the positive charge of the nanoparticles was observed. The $\mathrm{pKa}$ values of carboxylic and amino groups of AmpB were of 4.5 and 9.5, respectively, which is in accordance with Mazerski et al. ${ }^{53}$ Hence, in $\mathrm{pH} 5.0$, the carboxylic group did not presents charge, while the amino group presented a positive charge, giving like a final result a molecule with a slight positive charge. This finding may explain why no significant difference was observed between the zeta potential of the
NQC and NQC-AmpB nanoparticles. As a result, all the obtained nanoparticles had a positive charge. This finding is important, given that, in general, charged nanocarriers are more rapidly taken up by macrophages and other phagocytic cells, when compared with the uptake of nanoparticles with no charge. Further, positively charged nanoparticles accumulate in mononuclear phagocytes about twice more often as negatively charged carriers. ${ }^{13,54}$ In addition, Danesh-Bahreini et al described that positively charged nanoparticles are more quickly taken up by macrophages via phagocytosis than negatively charged or neutral nanoparticles. ${ }^{55}$ In our study, there was a high \%EE for the AmpB in the NQC-AmpB nanoparticles. Different ratios of Cs-ChS did not lead to significant differences in the $\% \mathrm{EE}$. This finding could be due to 
the concentration of $\mathrm{AmpB}$ in the nanoparticles, which was saturated in a 1:1 ratio. In contrast, at $\mathrm{pH} 5.0$, the amino group in Cs possesses a positive charge that interacts electrostatically with the sulfate group of $\mathrm{ChS}$, which possesses a negative charge, and the carboxylic group of AmpB, which possesses a negative charge, interacting electrostatically with a positive charge from the amino group of Cs.

It was also observed that TPP had an important function in the stability of the NQC nanoparticles, in that the use of TPP made the formulation stable for 3 weeks, while, without this compound, the formulation was not stable for a week (data not shown). A possible reason for this occurrence is that the TPP formed a highly cross-linked network structure in the nanoparticles. The limiting $\%$ EE factor is the solubility of AmpB in acidic media. In this study, a $\mathrm{pH}$ of nearly 2.0 was used with a $2 \%$ acetic acid solution to dilute the AmpB in aqueous media. ${ }^{41,42}$ So as to be successfully incorporated into the system, AmpB should be first solubilized, thus, in this study, a pH of 2.0 in aqueous media was used. This strategy was employed because a net charge on the molecule of AmpB, obtained in acid aqueous media, could increase the solubility in two ways: first, it could decrease the dimerization and association constants by ten-fold or more and, second, it could increase the threshold of degree of aggregation for which oligomers are soluble in water. For these reasons, Mazerski et al postulated that the presence of a net charge in the molecules is the main factor that induces the solubility of polyenes. ${ }^{53}$ In our study, an AmpB solution with the concentration of $586 \mu \mathrm{g} / \mathrm{mL}$ was found to be the maximum solubility obtained for this product, so this single dose was used with different proportions of $\mathrm{Cs}$ and $\mathrm{ChS}$ to analyze the \%EE and AmpB loading. The NQC-AmpB 1:1 formulation was found to have the smallest particle size, highest charge (positive zeta potential), and the best $\% \mathrm{EE}$ and AmpB loading profile.

ATR-FTIR analysis demonstrated that the sulfate group of ChS was linked with the ammonium group of Cs. No new absorption bands on the NQC-AmpB could be observed, which might mean that no obvious chemical reaction had occurred between AmpB and the delivery system. In a controlled drug-delivery system, an active product is incorporated into a polymeric network structure in such way that the drug will be released from the material in a slow and predefined manner. ${ }^{56,57}$ Depending on the drug delivery system and the application route, the release time may last from a few hours to several years. ${ }^{56}$ The lower release values obtained for the NQC-AmpB nanoparticles compared with pure AmpB were most likely to be due to strong interaction between the $\mathrm{AmpB}$ and the $\mathrm{Cs} / \mathrm{ChS}$ polymers. With this profile, it could be suggested that the reduced toxicity of AmpB in the NQC-AmpB formulation could also be attributed to the slow release of the drug incorporated in the nanoparticles inside the macrophages, favoring its anti-leishmanial activity for a longer time.

UV-Vis spectral analysis is useful for deducing aggregation of AmpB. UV-Vis spectral data led us to deduce that AmpB was present in NQC-AmpB nanoparticles in its monomeric form, and the lower intensity could be due to the small amount of available AmpB. Only this product has been reported to cause a change in the UV-Vis spectrum, with a very broad peak appearing around $329 \mathrm{~nm}$ and decreased intensities at 405, 364, and $382 \mathrm{~nm}$, when the monomeric state is converted to the aggregate state. ${ }^{58} \mathrm{~A}$ close association is believed to exist between AmpB's state of aggregation and its toxicity. ${ }^{58}$ The mechanism of action proposed for the toxic effect of this drug is derived from its interaction with sterols in bilayer membranes, such as cell walls, causing either the formation of pores in the membrane, leading to cellular destruction or to the inhibition of membrane repair. Monomeric AmpB associated with the sterols in fungal cell membranes, whereas self-associated AmpB could also form pores in cholesterol-containing membranes, leading to the drug's high toxicity to host cells. ${ }^{59}$

In the evaluation of anti-leishmanial activity, it was observed that Cs presented better anti-leishmanial activity in relation to the evaluated nanoparticles systems. A synergistic interaction between $\mathrm{Cs}$ and $\mathrm{ChS}$ was also observed against L. amazonensis and L. chagasi. The activity of NQC-AmpB nanoparticles was similar in comparison to the results visualized using pure AmpB; however, the cytotoxicity of the NQC-AmpB was approximately ten-fold lower than that of pure AmpB. In this context, the selectivity index of AmpB was increased ten-fold when added to the proposed delivery system in this study. In addition, pure $\mathrm{Cs}$ and $\mathrm{ChS}$, as well as NQ and NQC nanoparticles presented no significant toxicity for macrophages. Van de Ven et $\mathrm{al}^{60}$ and Corware et $\mathrm{al}^{61}$ also obtained blank nanoparticles without significant cytotoxicity in macrophages, but when they were loaded with $A m p B$, the evaluated systems demonstrated an increase in their toxicity. Finally, hemolytic activity was also determined as a cytotoxicity parameter, and it was observed that none of the nanoparticle formulations resulted in significant hemolysis.

\section{Conclusion}

The formulations tested in this study were found to have the desired characteristics of effective drug-delivery systems for use 
in the treatment of leishmaniasis. The formulations were able to reach the parasitic compartment in the infected cells, which is relevant mainly because a lower drug concentration will be required for administration in patients, which may, in turn, prevent the several toxic effects of the drugs that are often observed when conventional products are daily used at high doses.

As far as we are aware, the present study is the first to date to describe a new drug-delivery system based on NQC nanoparticles loaded with AmpB to treat leishmaniasis. This formulation, in addition to demonstrating effective antileishmanial activity, and a high capacity to treat infected macrophages, presented none of the toxic effects in mice or human cells that are normally observed when pure AmpB is administered. Thus, we could suggest that the NQC-AmpB formulation should be evaluated in future studies on in vivo anti-leishmanial activity, in an attempt to find new chemotherapeutic alternatives for the treatment of leishmaniasis.

\section{Acknowledgments}

The authors would like to thank Dr Manuel Soto (Centro de Biología Molecular Severo Ochoa [CSIC-UAM], Universidad Autónoma de Madrid, Spain) for scientific assistance. This work was supported by grants from Pró-Reitoria de Pesquisa from UFMG (Edital 03/2013), Instituto Nacional de Ciência e Tecnologia em Nano-biofarmacêutica (INCT-Nanobiofar), FAPEMIG (CBB-APQ-02364-08 and CBB-APQ-00496-11), and CNPq (APQ-472090/2011-9 and APQ-482976/2012-8). Miguel A Chávez-Fumagalli is a grant recipient of PNPD/ CAPES. Eduardo AF Coelho is a grant recipient of CNPq.

\section{Disclosure}

The authors report no conflicts of interest in this work.

\section{References}

1. Desjeux P. Leishmaniasis: current situation and new perspectives. Comp Immunol Microbiol Infect Dis. 2004;27(5):305-318.

2. World Health Organization (WHO). Control of the Leishmaniases: Report of a Meeting of the 399 WHO Expert Committee on the Control of Leishmaniases, Geneva, 22-26 March 2010. WHO 400 Technical Report Series 949. Geneva: WHO; 2010. Available from: whqlibdoc. who.int/trs/WHO_TRS_949_eng.pdf. Accessed December 18, 2013.

3. Alvar J, Vélez ID, Bern C, et al; WHO Leishmaniasis Control Team. Leishmaniasis worldwide and global estimates of its incidence. PloS One. 2012;7(5):e35671.

4. Croft SL, Coombs GH. Leishmaniasis - current chemotherapy and recent advances in the search for novel drugs. Trends Parasitol. 2003;19(11): 502-508.

5. Grevelink SA, Lerner EA. Leishmaniasis. J Am Acad Dermatol. 1996; 34(2):257-272.

6. Annaloro C, Olivares C, Usardi P, et al. Retrospective evaluation of amphotericin B deoxycholate toxicity in a single centre series of haematopoietic stem cell transplantation recipients. JAntimicrob Chemother. 2009;63(3):625-626.
7. Denning DW. Therapeutic outcome of invasive aspergillosis. Clin Infect Dis. 1996;23(3):608-615.

8. Bern C, Adler-Moore J, Berenguer J, et al. Liposomal amphotericin B for the treatment of visceral leishmaniasis. Clin Infect Dis. 2006;43(7): 917-924.

9. Egger SS, Meier S, Leu C, et al. Drug interactions and adverse events associated with antimycotic drugs used for invasive aspergillosis in hematopoietic SCT. Bone Marrow Transplant. 2009;45(7): 1197-1203.

10. Italia JL, Sharp A, Carter KC, Warn P, Kumar MN. Peroral amphotericin $\mathrm{B}$ polymer nanoparticles lead to comparable or superior in vivo antifungal activity to that of intravenous Ambisome ${ }^{\circledR}$ or Fungizone ${ }^{\mathrm{TM}}$. PLoS One. 2011;6(10):e25744.

11. Quintanar-Guerrero D, Allémann E, Fessi H, Doelker E. Preparation techniques and mechanisms of formation of biodegradable nanoparticles from preformed polymers. Drug Dev Ind Pharm. 1998;24(12):1113-1128.

12. de Carvalho RF, Ribeiro IF, Miranda-Vilela AL, et al. Leishmanicidal activity of amphotericin B encapsulated in PLGA-DMSA nanoparticles to treat cutaneous leishmaniasis in C57BL/6 mice. Exp Parasitol. 2013;135(2):217-222.

13. Asthana S, Jaiswal AK, Gupta PK, Pawar VK, Dube A, Chourasia MK. Immunoadjuvant chemotherapy of visceral leishmaniasis in hamsters using amphotericin B-encapsulated nanoemulsion template-based chitosan nanocapsules. Antimicrob Agents Chemother. 2013;57(4): $1714-1722$.

14. Shao K, Huang R, Li J, et al. Angiopep-2 modified PE-PEG based polymeric micelles for amphotericin B delivery targeted to the brain. J Control Release. 2010;147(1):118-126.

15. Yang ZL, Li XR, Yang KW, Liu Y. Amphotericin B-loaded poly(ethylene glycol)-poly(lactide) micelles: preparation, freeze-drying, and in vitro release. J Biomed Mater Res A. 2008;85(2):539-546.

16. Kreuter J. Nanoparticles. In: Swarbrick J, Boylan JC, editors. Encyclopedia of Pharmaceutical Technology. New York, NY: Marcel Dekker; 1988:165-190.

17. Janes KA, Fresneau MP, Marazuela A, Fabra A, Alonso MJ. Chitosan nanoparticles as delivery systems for doxorubicin. $J$ Control Release. 2001;73(2-3):255-267.

18. St John AL, Chan CY, Staats HF, Leong KW, Abraham SN. Synthetic mast-cell granules as adjuvants to promote and polarize immunity in lymph nodes. Nat Mater. 2012;11(3):250-257.

19. Huang L, Sui W, Wang Y, Jiao Q. Preparation of chitosan/chondroitin sulfate complex microcapsules and application in controlled release of 5-fluorouracil. Carbohydr Polym. 2010;80(1):168-173.

20. Ganza-GonzálezA, Anguiano-Igea S, Otero-Espinar FJ, Blanco Méndez J. Chitosan and chondroitin microspheres for oral-administration controlled release of metoclopramide. Eur J Pharm Biopharm. 1999;48(2):149-155.

21. Sui W, Huang L, Wang J, Bo Q. Preparation and properties of chitosan chondroitin sulfate complex microcapsules. Colloids Surf B Biointerfaces. 2008;65(1):69-73.

22. Yeh MK, Cheng KM, Hu CS, Huang YC, Young JJ. Novel protein-loaded chondroitin sulfate-chitosan nanoparticles: preparation and characterization. Acta Biomater. 2011;7(10):3804-3812.

23. Hu CS, Chiang CH, Hong PD, Yeh MK. Influence of charge on FITCBSA-loaded chondroitin sulfate-chitosan nanoparticles upon cell uptake in human Caco-2 cell monolayers. Int J Nanomedicine. 2012;7: 4861-4872.

24. Tsai HY, Chiu CC, Lin PC, Chen SH, Huang SJ, Wang LF. Antitumor efficacy of doxorubicin released from crosslinked nanoparticulate chondroitin sulfate/chitosan polyelectrolyte complexes. Macromol Biosci. 2011;11(5):680-688.

25. Bhattarai N, Gunn J, Zhang M. Chitosan-based hydrogels for controlled, localized drug delivery. Adv Drug Deliv Rev. 2010;62(1):83-99.

26. Thongngam M, McClements DJ. Influence of $\mathrm{pH}$, ionic strength, and temperature on self-association and interactions of sodium dodecyl-sulfate in the absence and presence of chitosan. Langmuir. 2005;21(1):79-86. 
27. Iovu M, Dumais G, du Souich P. Anti-inflammatory activity of chondroitin sulfate. Osteoarthritis Cartilage. 2008;16 Suppl 3:S14-S18.

28. Ronca F, Palmieri L, Panicucci P, Ronca G. Anti-inflammatory activity of chondroitin sulfate. Osteoarthritis Cartilage. 1998;6 Suppl A:14-21.

29. Ronca F, Palmieri L, Panicucci P, Ronca G. Anti-inflammatory activity of chondroitin sulfate. Osteoarthritis Cartigale. 1998;6(A):14-21.

30. Kirker KR, LuoY, Morris SE, Shelby J, Prestwich GD. Glycosaminoglycan hydrogels as supplemental wound dressings for donor sites. J Burn Care Rehab. 2004;25(3):276-286.

31. Kirker KR, LuoY, Nielson JH, Shelby J, Prestwich GD. Glycosaminoglycan hydrogel films as bio-interactive dressings for wound healing. Biomaterials. 2002;23(17):3661-3671.

32. Wang LF, Wang JM, Chiang YL. Insolubilization of sodium chondroitin sulfate by forming a semi-interpenetrating polymer network with acrylic acid: a potential carrier for colon-specific drug delivery. J Appl Polym Sci. 2002;85(1):114-122.

33. Sintov A, Di-Capua N, Rubinstein A. Cross-linked chondroitin sulphate: characterization for drug delivery purposes. Biomaterials. 1995;16(6):473-478.

34. Costa DF, Franca JR, Ribeiro TG, Kaplan MA, Faraco AA, Castilho RO. Development and characterization of polymeric nanoparticles as Barbatimão (Stryphnodendron obovatum) standardized fraction carrier. Adv Biosci Biotechnol. 2012;4(1):89-92.

35. Calvo P, Remuñán-López C, Vila-Jato JL, Alonso MJ. Novel hydrophilic chitosan-polyethylene oxide nanoparticles as protein carriers. J Appl Polym Sci. 1997;63(1):125-132.

36. Grenha A, Gomes ME, Rodrigues M, et al. Development of new chitosan/carrageenan nanoparticles for drug delivery applications. J Biomed Mater Res A. 2010;92(4):1265-1272.

37. Müller RH, Schmidt S, Buttle I, Akkar A, Schmitt J, Brömer S. SolEmuls-novel technology for the formulation of iv emulsions with poorly soluble drugs. Int J Pharm. 2004;269(2):293-302.

38. Moreno MA, Frutos P, Ballesteros MP. Lyophilized lecithin based oil-water microemulsions as a new and low toxic delivery system for amphotericin B. Pharm Res. 2001;18(3):344-351.

39. Santos CM, Oliveira RB, Arantes VT, et al. Amphotericin B-loaded nanocarriers for topical treatment of cutaneous leishmaniasis: development, characterization, and in vitro skin permeation studies. J Biomed Nanotechnol. 2012;8(2):322-329.

40. Nahar M, Jain NK. Preparation, characterization and evaluation of targeting potential of amphotericin B-loaded engineered PLGA nanoparticles. Pharm Res. 2009;26(12):2588-2598.

41. Coelho EA, Tavares CA, Carvalho FA, et al. Immune responses induced by the Leishmania (Leishmania) donovani A2 antigen, but not by the LACK antigen, are protective against experimental Leishmania (Leishmania) amazonensis infection. Infect Immun. 2003;71(7):3988-3994.

42. Valadares DG, Duarte MC, Oliveira JS, et al. Leishmanicidal activity of the Agaricus blazei Murill in different Leishmania species. Parasitol Int. 2011;60(4):357-363.

43. Fumarola L, Spinelli R, Brandonisio O. In vitro assays for evaluation of drug activity against Leishmania spp. Res Microbiol. 2004;155(4): 224-230.

44. Löfgren SE, Miletti LC, Steindel M, Bachère E, Barracco MA. Trypanocidal and leishmanicidal activities of different antimicrobial peptides (AMPs) isolated from aquatic animals. Exp Parasitol. 2008;118(2):197-202.
45. Jung SH, Lim DH, Jung SH, et al. Amphotericin B-entrapping lipid nanoparticles and their in vitro and in vivo characteristics. Eur J Pharm Sci. 2009;37(3-4):313-320.

46. Onishi H, Machida Y. Biodegradation and distribution of water-soluble chitosan in mice. Biomaterials. 1999;20(2):175-182.

47. Huang M, Ma Z, Khor E, Lim LY. Uptake of FITC-chitosan nanoparticles by A549 cells. Pharm Res. 2002;19(10):1488-1494.

48. Adams ML, Kwon GS. Relative aggregation state and hemolytic activity of amphotericin B encapsulated by poly(ethylene oxide)-block-poly(Nhexyl-L-aspartamide)-acyl conjugate micelles: effects of acyl chain length. $J$ Control Release. 2003;87(1-3):23-32.

49. Tasdemir D, Kaiser M, Brun R, et al. Antitrypanosomal and antileishmanial activities of flavonoids and their analogues: in vitro, in vivo, structure-activity relationship, and quantitative structureactivity relationship studies. Antimicrob Agents Chemother. 2006;50(4): 1352-1364.

50. Pujals G, Suñé-Negre JM, Pérez P, et al. In vitro evaluation of the effectiveness and cytotoxicity of meglumine antimoniate microspheres produced by spray drying against Leishmania infantum. Parasitol Res. 2008;102(6):1243-1247.

51. Nakajima A, Shinoda K. Complex formation between oppositely charged polysaccharides. J Colloid Interface Sci. 1976;55(1):126-132.

52. Chen WB, Wang LF, Chen JS, Fan SY. Characterization of polyelectrolyte complexes between chondroitin sulfate and chitosan in the solid state. J Biomed Mater Res A. 2005;75(1):128-137.

53. Mazerski J, Grzybowska J, Borowski E. Influence of net charge on the aggregation and solubility behaviour of amphotericin B and its derivatives in aqueous media. Eur Biophys J. 1990;18(3):159-164.

54. Batrakova EV, Gendelman HE, Kabanov AV. Cell-mediated drug delivery. Expert Opin Drug Deliv. 2011;8(4):415-433.

55. Danesh-Bahreini MA, Shokri J, Samiei A, Kamali-Sarvestani E, Barzegar-Jalali M, Mohammadi-Samani S. Nanovaccine for leishmaniasis: preparation of chitosan nanoparticles containing Leishmania superoxide dismutase and evaluation of its immunogenicity in BALB/c mice. Int J Nanomedicine. 2011;6:835-842.

56. Wise DL, editor. Handbook of Pharmacentical Controlled Release Technology. New York, NY: Marcel Dekker, 2000; Chap 9: pp 183-210.

57. Jogani V, Jinturkar K, Vyas T, Misra A. Recent patents review on intranasal administration for CNS drug delivery. Recent Pat Drug Deliv Formul. 2008;2(1):25-40.

58. Legrand P, Romero EA, Cohen BE, Bolard J. Effects of aggregation and solvent on the toxicity of amphotericin B to human erythrocytes. Antimicrob Agents Chemother. 1992;36(11):2518-2522.

59. Darole PS, Hegde DD, Nair HA. Formulation and evaluation of microemulsion based delivery system for amphotericin B. AAPS Pharm Sci Tech. 2008;9(1):122-128.

60. Van de Ven H, Paulussen C, Feijens PB, et al. PLGA nanoparticles and nanosuspensions with amphotericin B: Potent in vitro and in vivo alternatives to Fungizone and AmBisome. J Control Release. 2012;161(3): 795-803.

61. Corware K, Harris D, Teo I, et al. Accelerated healing of cutaneous leishmaniasis in non-healing $\mathrm{BALB} / \mathrm{c}$ mice using water soluble amphotericin B-polymethacrylic acid. Biomaterials. 2011;2(31): 8029-8039.
International Journal of Nanomedicine

\section{Publish your work in this journal}

The International Journal of Nanomedicine is an international, peerreviewed journal focusing on the application of nanotechnology in diagnostics, therapeutics, and drug delivery systems throughout the biomedical field. This journal is indexed on PubMed Central, MedLine, CAS, SciSearch ${ }^{\circledR}$, Current Contents ${ }^{\circledR} /$ Clinical Medicine,
Dovepress

Journal Citation Reports/Science Edition, EMBase, Scopus and the Elsevier Bibliographic databases. The manuscript management system is completely online and includes a very quick and fair peer-review system, which is all easy to use. Visit http://www.dovepress.com/ testimonials.php to read real quotes from published authors. 